\title{
Experimental and Theoretical Investigation of the Proton-Bound Dimer of Lysine
}

\author{
Ronghu Wu, ${ }^{1,2}$ Richard A. Marta, ${ }^{1}$ Jonathan K. Martens, ${ }^{1}$ Kris R. Eldridge, ${ }^{1}$ \\ Terry B. McMahon ${ }^{1}$ \\ ${ }^{1}$ Department of Chemistry, University of Waterloo, Waterloo, Ontario, Canada N2L 3G1 \\ ${ }^{2}$ Harvard Medical School, 240 Longwood Ave., Boston, MA, 02115, USA
}

\begin{abstract}
The structure of the proton-bound lysine dimer has been investigated by infrared multiple photon dissociation (IRMPD) spectroscopy and electronic structure calculations. The structures of different possible isomers of the proton-bound lysine dimer have been optimized at the B3LYP/ $6-31+G(d)$ level of theory and IR spectra calculated using the same computational method. Based on relative Gibbs free energies (298 K) calculated at the MP2/aug-cc-pVTZ//B3LYP/6-31 $+\mathrm{G}(\mathrm{d})$ level of theory, LL-CS01, and followed closely $\left(1.1 \mathrm{~kJ} \mathrm{~mol}^{-1}\right)$ by LL-CS02 are the most stable non-zwitterionic isomers. At the MP2/aug-cc-pVTZ//6-31+G(d) and MP2/aug-cc-pVTZ//6$31+(\mathrm{d}, \mathrm{p})$ levels of theory, isomer LL-CSO2 is favored by 3.0 and $2.3 \mathrm{~kJ} \mathrm{~mol}^{-1}$, respectively. The relative Gibbs free energies calculated by the aforementioned levels of theory for LL-CS01 and LL-CS02 are very close and strongly suggest that diagnostic vibrational signatures found in the IRMPD spectrum of the proton-bound dimer of lysine can be attributed to the existence of both isomers. LL-ZW01 is the most stable zwitterionic isomer, in which the zwitterionic structure of the neutral lysine is well stabilized by the protonated lysine moiety via a very strong intermolecular hydrogen bond. At the MP2/aug-cc-pVTZ//B3LYP/6-31+G(d), MP2/aug-cc-pVTZ//6-31+G(d) and MP2/aug-cc-pVTZ//6-31+G(d,p) levels of theory, the most stable zwitterionic isomer (LLZW01) is less favored than LL-CS01 by 7.3, 4.1 and $2.3 \mathrm{~kJ} \mathrm{~mol}^{-1}$, respectively. The experimental IRMPD spectrum also confirms that the proton-bound dimer of lysine largely exists as charge-solvated isomers. Investigation of zwitterionic and charge-solvated species of amino acids in the gas phase will aid in a further understanding of structure, property, and function of biological molecules.
\end{abstract}

Key words: Proton-bound lysine dimer, Infrared multiple photon dissociation (IRMPD), Electronic structure calculations, Vibrational spectra, Charge solvated structure, Zwitterionic gas phase structure

\section{Introduction}

Z witterionic structures exist extensively in biological systems, and are directly related to the function and activity of amino acids, peptides, and proteins [1, 2]. Naturally occurring amino acids are well known to exist as zwitterions in the solid state and aqueous solution within a wide range of $\mathrm{pH}$ values, whereas in the gas phase the

Correspondence to: Terry McMahon; e-mail: mcmahon@uwaterloo.ca isolated amino acids are exclusively in the canonical form. However, interactions with other molecules or ions can play a key role in stabilizing the zwitterionic form of amino acids, as demonstrated by many previous studies [3-17].

Stabilization of zwitterionic structures of a series of amino acids (Gly, Ala, Val, Ser, and Pro) by various ammonium ions has been investigated systematically [18] and some generalizations with respect to the relative stability of zwitterionic structures may be drawn. First, for the complexes of aliphatic amino acids (Gly, Ala, and Val) with amines, as the proton affinity (PA) of the amino 
acid increases, the zwitterionic structure becomes more energetically favorable relative to the non-zwitterionic isomer. Second, as the PA of the amine increases, the zwitterionic structure of a given amino acid within the complex becomes gradually less favorable. Thirdly, compared with the other amino acids, Pro, the only secondary amine among the 20 naturally occurring amino acids, has a much more pronounced tendency to form a zwitterionic structure. Our previous high pressure mass spectrometric and infrared multiple photon dissociation (IRMPD) spectroscopic results confirmed that the zwitterionic structure of proline can be stabilized by a protonated methylamine, [19] and that a zwitterionic structure also exists in the proton-bound proline dimer [20]. IRMPD experimental results also confirmed that zwitterionic proline may be stabilized by $\mathrm{Na}^{+}[21]$.

Lysine is one of the more basic naturally occurring amino acids because it has a primary amine side chain, which is considerably important for its structure and function. The existence of a side chain makes the situation more complicated because the side chain not only has marked effects on the proton affinity and gas basicity of the corresponding amino acid, but also can interact with other species. According to the above trend, lysine would be predicted to form a zwitterionic structure relatively easily. The stabilization of the zwitterionic structure of lysine by different metal ions has also been investigated in the literature [22, 23]. The IRMPD experimental results indicated that lysine is non-zwitterionic in complexes with either $\mathrm{Li}^{+}, \mathrm{Na}^{+}$or $\mathrm{K}^{+}$[22]. The lowest-energy structure of lithiated lysine without a water molecule is non-zwitterionic and the metal ion interacts with both nitrogen atoms and the carbonyl oxygen. Structures in which lysine is zwitterionic are higher in energy by more than $29 \mathrm{~kJ} \mathrm{~mol}^{-1}$ than the most stable isomer. In contrast, the singly hydrated clusters with both zwitterionic and non-zwitterionic forms of lysine are more similar in energy, with the non-zwitterionic form more stable by only $7.1 \mathrm{~kJ} \mathrm{~mol}^{-1}$ [23]. Computational results have indicated that the interaction between lysine and $\mathrm{H}_{2} \mathrm{O}$ significantly affects the relative conformational stabilities and a single water molecule is sufficient to produce the stable zwitterionic structures in the gas phase [24]. It is thus very interesting to investigate the structure of the proton-bound lysine dimer in the gas phase and address the question of whether a zwitterionic structure exists in the dimer.

In the present work, the structures of the proton-bound dimer of lysine $\left[(\text { Lys })_{2} \mathrm{H}^{+}\right]$have been investigated using mass-selected IRMPD spectroscopy and electronic structure calculations. The structures of many different isomers have been optimized at the B3LYP/6-31+G(d) level of theory. The gas phase IRMPD spectrum of $(\mathrm{Lys})_{2} \mathrm{H}^{+}$has been recorded and compared with the computed IR spectra. The stabilization of possible zwitterionic structures of lysine has been investigated.

\section{Experimental and Electronic Structure Calculations IRMPD Experimental}

Experiments have been carried out using the Free Electron Laser (FEL) at the Centre de Laser Infrarouge d'Orsay (CLIO) facility in Orsay (France) coupled to an electrospray ionization-ion trap mass spectrometer (Bruker Esquire3000+, 40 Manning Road, Billerica, MA, USA 01821). This experimental configuration has been described in detail previously [25-27]. The FEL facility is based on emission from a 10$50 \mathrm{MeV}$ electron beam. For the high energy electron beam, the emission photon wavelength can be tuned by adjusting the gap of an undulator, which is placed in the optical cavity. In the present work, the electron energy was set to $48 \mathrm{MeV}$ to continuously scan from $1000 \mathrm{~cm}^{-1}$ to $2000 \mathrm{~cm}^{-1}$. The IR-FEL output consists of macropulses $8 \mu$ s in length with a repetition rate of $25 \mathrm{~Hz}$. Each macropulse involves approximately 500 micropulses with a width of a few picoseconds. For a typical average IR power of $500 \mathrm{~mW}$, the corresponding micropulse and macropulse energies are about $40 \mu \mathrm{J}$ and $20 \mathrm{~mJ}$, respectively.

In order to produce the ion of $(\mathrm{Lys})_{2} \mathrm{H}^{+}$, a solution of lysine dissolved in a mixture of water and methanol, with a very small amount of formic acid, was used. The solution (concentration of $10^{-6}$ to $10^{-5} \mathrm{M}$ ) was introduced into the mass spectrometer using electrospray ionization, and the desired ionic species was isolated and confined in the ion trap. The IR-FEL beam with a desired wavelength was focused and introduced into the center of the ion trap of the mass spectrometer. Mass spectra were recorded following irradiation and ten mass spectra were accumulated at each wavelength. IRMPD spectra were obtained by scanning the wavelength in steps of $\sim 4 \mathrm{~cm}^{-1}$. The spectra reported here are expressed as the fragmentation efficiency, $P_{\text {frag, }}$, as a function of the photon energy, in $\mathrm{cm}^{-1}$.

$$
\mathrm{P}_{\text {frag }}=-\log \left(\frac{\mathrm{I}_{\text {parent }}}{\sum \mathrm{I}_{\text {fragment }}+\mathrm{I}_{\text {parent }}}\right)
$$

\section{Electronic Structure Calculations}

In order to generate candidate structures for the lysine proton-bound dimer, Replica-Exchange Molecular Dynamics (REMD) simulations were performed combining the polarizable AMOEBA force field and the Tinker molecular dynamics package $[28,29]$. This approach has previously been successfully used in structural searching and potential energy surface mapping of charged biological molecules [30]. Twelve trajectories were used over a temperature range of $200-600 \mathrm{~K}$, with 500 exchanges and $1.0 \mathrm{fs}$ steps for a total time of $5.0 \mathrm{~ns}$. This protocol produced more than 800 structures. The 10 best candidate structures were then subjected to high level electronic structure calculations. These calculations have been performed using the Gaussian 09 software package [31]. 
The structures of all proposed isomers of the cluster of (Lys) ${ }_{2} \mathrm{H}^{+}$were optimized at the density functional theory (DFT) level, employing the B3LYP exchange-correlation functional and the $6-31+\mathrm{G}(\mathrm{d})$ basis set. B3LYP is a relatively reliable and economical computational method and is extensively employed in the investigation of biological molecules [32-38].

Geometries for the most relevant species obtained using the B3LYP/6-31+G(d) level of theory have been recalculated at the MP2/6-31+g(d) and MP2/6-31+G(d,p) protocols. This was done in order to investigate the possibility of large differences in conformation existing between structures calculated by the different methods described above. For the most stable isomers of $(\text { Lys })_{2} \mathrm{H}^{+}$ the mean absolute deviation (MAD) between all calculated bond lengths at the B3LYP/6-31+G(d) and MP2/6$31+\mathrm{G}(\mathrm{d})$ levels of theory is found to be only $0.016 \AA$, or $1.2 \%$ of the average molecular bond length. The MAD between all calculated bond lengths at the B3LYP/6-31+ $\mathrm{G}(\mathrm{d})$ and MP2/6-31+G(d,p) levels of theory is found to be only $0.019 \AA$, or $1.4 \%$ of the average molecular bond length. These observations would suggest that B3LYP/6$31+\mathrm{G}(\mathrm{d})$ performs similarly as the MP $2 / 6-31+\mathrm{G}(\mathrm{d})$ and MP2/6-31+(d,p) levels of theory for the purpose of obtaining optimized geometries of $(\text { Lys })_{2} \mathrm{H}^{+}$, which is also consistent with what is found for optimizations done at higher levels of theory on similar sized systems that we have described recently $[37,38]$. This comparison is very useful since optimizations and notably, frequencies calculated using the MP2 method are considerably more costly than those obtained with B3LYP coupled with equivalent sized basis sets.

It has been well established that hybrid DFT methods such as B3LYP can outperform local or gradient-corrected DFT, and MP2 for the prediction of fundamental frequencies and their associated infrared intensities. Fundamental frequencies calculated by the MP2 method are significantly more costly, and in many cases, less accurate than those produced by B3LYP; thus, all geometry optimizations and calculated frequencies compared here will be determined using the B3LYP method [21, 39-47]. Anharmonic frequencies $[31,48]$ have also been obtained at the B3LYP/6-31+G (d,) level of theory for the most chemically significant species.

In order to obtain a more accurate interpretation of longrange interactions, single point energies have been calculated using a MP2/aug-cc-pVTZ protocol since energies obtained at the MP2 method for hydrogen bonding are more accurate in almost all cases [49]. More specifically, this is true when the MP2 method is coupled to a large basis set such as, augcc-pVTZ [50]. Zero-point energy and thermal energy corrections at $298 \mathrm{~K}$ were also considered. The relative entropy changes were obtained from the B3LYP/6-31+G(d) level of theory. The relative calculated thermochemical values for the proposed isomers at different levels of theory are summarized in Table 1.
Table 1. Relative Thermochemical Values for the Proposed Lowest Energy Isomers of the Protonated Lysine Dimer Calculated at the MP2/aug-ccpVTZ//B3LYP/6-31+G(d) ${ }^{\text {a }}$, MP2/aug-cc-pVTZ//6-31+G(d) ${ }^{\text {b }}$, and MP2/ aug-cc-pVTZ//6-31+G(d,p) $)^{\mathrm{c}}$ Levels of Theory

\begin{tabular}{lcccc}
\hline Isomer & $\begin{array}{l}\text { Level of } \\
\text { theory }\end{array}$ & $\begin{array}{l}\Delta \Delta \mathrm{H}_{298}{ }^{\circ} \\
\left(\mathrm{kJ} \mathrm{mol}^{-1}\right)\end{array}$ & $\begin{array}{l}\Delta \Delta \mathrm{S}_{298}{ }^{\circ} \\
\left(\mathrm{J} \mathrm{mol}^{-1} \mathrm{~K}^{-1}\right)\end{array}$ & $\begin{array}{l}\Delta \Delta \mathrm{G}_{298}{ }^{\circ} \\
\left(\mathrm{kJ} \mathrm{mol}^{-1}\right)\end{array}$ \\
\hline LL-CS01 & $\mathrm{a}$ & 0 & 0 & 0 \\
& $\mathrm{~b}$ & 0 & 0 & 0 \\
LL-CS02 & $\mathrm{c}$ & 0 & 0 & 0 \\
& $\mathrm{a}$ & 0.5 & -1.9 & 1.1 \\
& $\mathrm{~b}$ & -2.7 & 0.8 & -3.0 \\
LL-ZW01 & $\mathrm{c}$ & -2.4 & -0.3 & -2.3 \\
& $\mathrm{a}$ & 6.2 & -3.7 & 7.3 \\
LL-ZW02 & $\mathrm{b}$ & 6.9 & 9.4 & 4.1 \\
& $\mathrm{c}$ & 4.7 & 7.8 & 2.3 \\
& $\mathrm{a}$ & 2.2 & -33.4 & 12.2 \\
LL-CS03 & $\mathrm{b}$ & 1.0 & -22.1 & 7.5 \\
& $\mathrm{c}$ & -1.6 & -24.5 & 5.7 \\
& $\mathrm{a}$ & -0.8 & -42.2 & 11.8 \\
& $\mathrm{~b}$ & -0.1 & -38.0 & 11.2 \\
& $\mathrm{c}$ & -1.2 & -41.4 & 11.1 \\
\hline
\end{tabular}

\section{Results and Discussion}

Using the experimental IRMPD spectroscopic and computational data, the structure of the proton-bound lysine dimer has been elucidated. The results demonstrate unambiguously that in the proton-bound dimer, the three ammonium ion hydrogens of the side chain-protonated lysine participate in three hydrogen bonds. One of these is an intramolecular hydrogen bond with the carbonyl oxygen of the protonated lysine moiety. Simultaneously, the two other ammonium ion hydrogens form hydrogen bonds with both the side chain amino group and the carbonyl oxygen of the neutral monomer moiety (Figure 1; LL-CS01 and LL-CS02).

Many isomers of the proton-bound lysine dimer have been optimized and the five most stable structures are shown in Figure 1, with their calculated thermochemical values being summarized in Table 1. According to the results of calculations at the MP2/aug-cc-pVTZ//B3LYP/6-31+G(d) level of theory, LL-CS01 and LL-CS02 are the most stable isomers, with LL-CS01 predicted to be favored by only $1.1 \mathrm{~kJ} \mathrm{~mol}^{-1}$ in Gibbs free energy over LL-CS02. In LL$\mathrm{CS} 01$, the proton is bound to the side chain amino group of one lysine, as in the protonated lysine monomer, followed by the formation of one intramolecular hydrogen bond with the $\alpha$-amino nitrogen and two intermolecular hydrogen bonds with the carbonyl oxygen and side chain amino nitrogen of the neutral lysine moiety, respectively, with almost identical bond lengths of $1.88,1.85$, and $1.89 \AA$. In addition, two intramolecular hydrogen bonds exist in the proton-bound dimer. In LL-CSO2, all interactions are analogous to those found in LL-CS01; however, in this case, an intramolecular hydrogen bond is formed by the carbonyl oxygen of the protonated lysine monomer. The bond lengths of LL-CS02 similar to those of LL-CS01 described above are 1.89, 1.86, and 1.88 $\AA$. At the MP2/augcc-pVTZ//6-31+G(d) and MP2/aug-cc-pVTZ//6-31+(d,p) levels of theory, isomer LL-CS02 is favored by 3.0 and $2.3 \mathrm{~kJ} \mathrm{~mol}^{-1}$, respectively. The absolute enthalpy of binding 


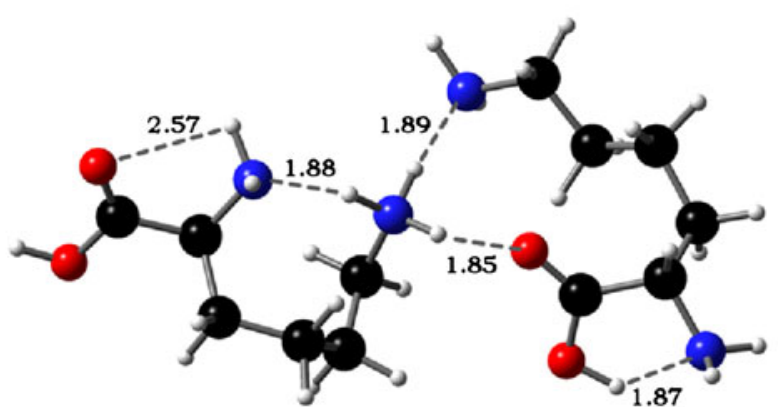

LL-CS01

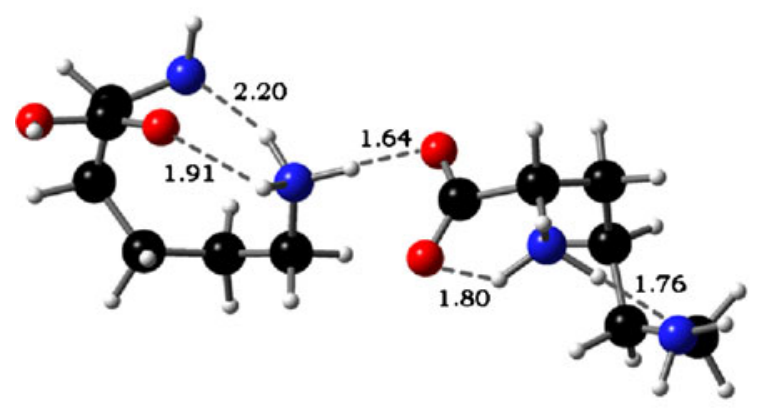

LL-ZW01 carbon

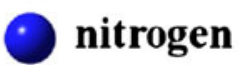

oxygen

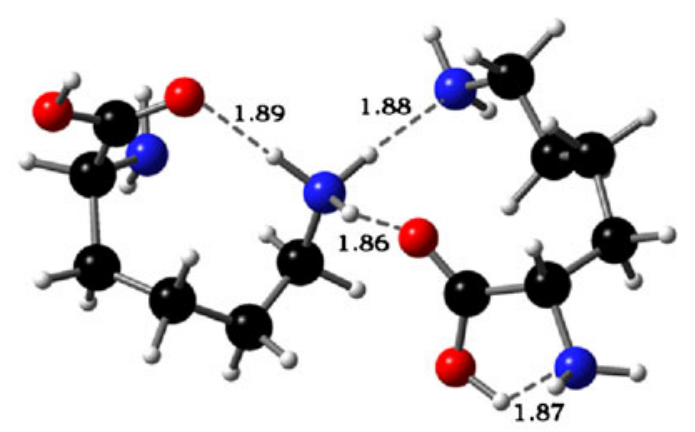

LL-CS02

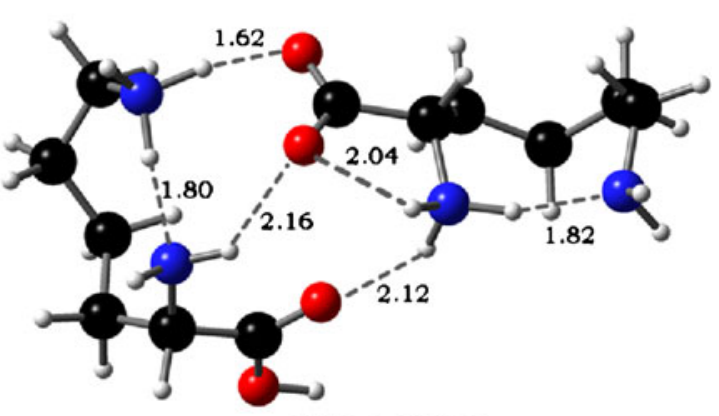

LL-ZW02

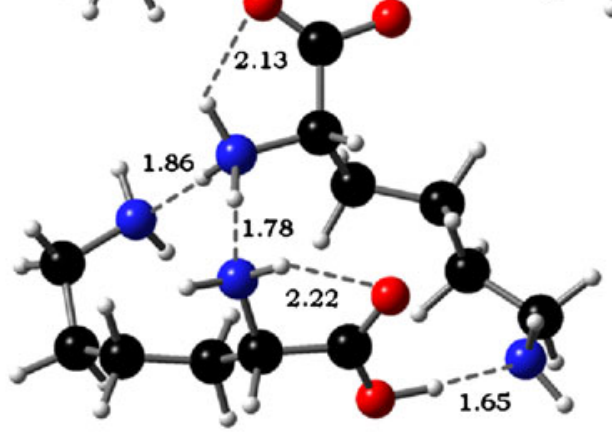

\section{LL-CS03}

Figure 1. Structures of the five most stable isomers of the lysine proton-bound dimer obtained at the B3LYP/6-31+G(d) level of theory. Bond lengths are reported in units of Angstroms ( $(\AA)$

between neutral and protonated lysine is $126 \mathrm{~kJ} \mathrm{~mol}^{-1}$ at the MP2/aug-cc-pVTZ//B3LYP/6-31+G(d) level of theory. The proton is bound to the amino nitrogen of the side chain, followed by the formation of two intramolecular hydrogen bonds with the $\alpha$-amino nitrogen and carbonyl oxygen with bond lengths of 1.91 and $2.20 \AA$, respectively. Compared with the bond lengths in the protonated lysine monomer of 1.80 and $1.91 \AA$ (obtained by the same computational method, B3LYP/6-31+G(d)), these bond lengths are markedly longer because the protonated amino group forms another very strong intermolecular hydrogen bond with the carboxylate group of the neutral zwitterionic lysine with a very short bond length of $1.64 \AA$. In the zwitterionic lysine moiety, the protonated $\alpha$-amino group forms two strong intramolecular hydrogen bonds with the adjacent carboxylate and side chain amino group, respectively. Thus, the negatively charged carboxylate group may effectively be stabilized by the two positively charged protonated amino groups. When the proton is placed at the carboxylate group as an input structure, it spontaneously transfers to the amino group of the other lysine without a barrier.

The structure of the second most stable zwitterionic isomer, LL-ZW02, is notably different from that of LLZW01. Multiple intermolecular hydrogen bonds exist between the protonated and neutral lysines. The proton is also bound to the amino nitrogen of the side chain, followed by the formation of an intramolecular hydrogen bond with the $\alpha$-amino nitrogen and an intermolecular hydrogen bond with one of the two oxygen atoms of the carboxylate group with a very short bond length of only $1.62 \AA$. The other oxygen of the carboxylate group forms an intramolecular hydrogen bond with the adjacent protonated amino group and an intermolecular hydrogen bond with the $\alpha$-amino group of the protonated lysine, respectively. These two hydrogen bonds are relatively weak with bond lengths of 2.04 and 2.16 $\AA$. Compared with LL-ZW01, the zwitterionic moiety of LL-ZW02 is very similar, however, the structures of the protonated lysine moieties are totally different. At the 
MP2/aug-cc-pVTZ//B3LYP/6-31+G(d) level of theory, LLZW02 is favored by $4.0 \mathrm{~kJ} \mathrm{~mol}^{-1}$ in enthalpy than LL-ZW01 $\checkmark$ at $298 \mathrm{~K}$; however, since the structure of LL-ZW02 is significantly tighter it is less favored $\left(30 \mathrm{Jmol}^{-1} \mathrm{~K}^{-1}\right)$ than LL-ZW01 entropically; thus, LL-ZW01 is favored in Gibbs free energy. It follows that the structure of LL-ZW01 is also favored over LL-ZW02 at the MP2/aug-cc-pVTZ//6-31+G (d) and MP2/aug-cc-pVTZ//6-31+G(d,p) levels of theory; however, the relative difference in Gibbs free energy between the isomers decreases, respectively.

In LL-CS03 a proton is bound to the $\alpha$-amino group and the protonated amino group, acting as a proton-donor, forms two strong intermolecular hydrogen bonds with the two amino groups of the neutral lysine, respectively, and an intramolecular hydrogen bond with the adjacent carbonyl oxygen. The carboxylic acid group in the neutral lysine moiety acts as a proton-donor to form a very strong hydrogen bond with the side chain amino group in the protonated lysine moiety with a bond length of $1.65 \AA$. As for LL-ZW02, this isomer also has a very tight structure, which results in it being entropically less favorable. LL$\mathrm{CS} 03$ is $0.8 \mathrm{~kJ} \mathrm{~mol}^{-1}$ lower in enthalpy than LL-CS01 at the MP2/aug-cc-pVTZ//B3LYP/6-31+G(d) level of theory. Considering the entropy contribution, LL-CS03 is $12 \mathrm{~kJ} \mathrm{~mol}^{-1}$ higher in Gibbs free energy than LL-CS01. The difference is that the proton is bound to the side chain amino group. Its free energy is even higher than that of LLCS03.

In order to elucidate the structure of the proton-bound lysine dimer, the IRMPD spectrum of the proton-bound lysine dimer has been recorded and shown in Figure 2, together with the computed spectra for the three most stable isomers. Two calculated spectra have been obtained at the B3LYP/6-31+G (d) level of theory, with Figure $2 \mathrm{a}$ obtained using an anharmonic approximation [31, 48] and Figure $2 \mathrm{~b}$ using harmonic frequencies scaled by a factor of 0.96 [51]. In addition, the spectrum in Figure $2 \mathrm{c}$ is calculated at the MP2/6$31+\mathrm{G}(\mathrm{d})$ level of theory using a harmonic approximation scaled by 0.95 [51]. The calculated band frequencies and intensities were convoluted, assuming a Lorentzian profile with a $15 \mathrm{~cm}^{-1}$ full-width at half-maximum, in order to take into account the rotational bandwidth and the dynamics of the multiphoton excitation [52]. Both the anharmonic and scaled harmonic B3LYP/6-31+G(d) spectra shown in Figure 2 match more convincingly with the IRMPD spectrum than the scaled harmonic MP2/6-31+G(d) spectrum; thus the B3LYP/6-31+G (d) anharmonic values are used as the basis of comparison between calculated and experimental values.

The IRMPD spectrum of the proton-bound lysine dimer has been obtained and is shown in Figure 2. Following mass-isolation and IRMPD, the exclusive fragment ion is protonated lysine because the noncovalent interaction between neutral lysine and protonated lysine is much weaker than the covalent bonds found in the dimer.

The experimental IRMPD spectrum may often be inconsistent with the calculated spectrum [19, 20, 52-54]. This may be the result of one or more possible factors. First, the multiple photon excitation process may not precisely mimic the calculated single photon absorption spectrum. Second, the calculated IR spectra may themselves be subject to some error, particularly, for modes involving hydrogen bonds. Third, the IRMPD spectrum is a function not only of the absorption spectrum, but also the fragmentation efficiency of the ion. In addition, the laser intensity is not constant in the range of the experimental wavelength and can fluctuate by an average of $\pm 15 \%$ over the experimental range of 1000 to $1800 \mathrm{~cm}^{-1}$; apparently, this has an effect on the band intensities of the experimental spectrum.

An examination of the current experimental IRMPD spectra of the lysine proton-bound dimer and the calculated spectra of the three most stable isomers indicate that the experimental spectra fit very well with those for the two most stable isomers, LL-CSO1 and LL-CS02, i.e. nonzwitterionic isomers.

In the experimental IRMPD spectra, the strong band at $1396 \mathrm{~cm}^{-1}$ can be assigned to the bending vibration of hydrogen-bonded hydroxyl groups in LL-CS01 and LLCS02, which is consistent with the calculated anharmonic vibrations (B3LYP/6-31+G(d)) of 1396 and $1390 \mathrm{~cm}^{-1}$, respectively. Another strong band at $1756 \mathrm{~cm}^{-1}$ is apparently due to the stretch $(v)$ of the carbonyl group. In the calculated anharmonic spectrum of LL-CS01 there exists a carboxyl hydrogen bend $(\delta)$, in conjunction with a carbonyl stretch $(v)$ for each lysine monomer, at 1790 and $1747 \mathrm{~cm}^{-1}$, which appear as separated bands in the simulated spectrum. For LL-CS02, the two bands at 1752 and $1747 \mathrm{~cm}^{-1}$ merge into one broad band in the simulated spectrum with a $15 \mathrm{~cm}^{-1}$ full-width at half-maximum. However, the resolution of the IRMPD spectra is not sufficient to reproduce this and therefore only one relatively broad band appears in this region. A weak broad band at around $1603 \mathrm{~cm}^{-1}$ is due to the symmetric bending vibrations of the $-\mathrm{NH}_{3}$ groups and the symmetric bending modes of the $-\mathrm{NH}_{2}$ groups in LLCS01 and LL-CS02.

For the calculated spectrum of the most stable zwitterionic isomer, LL-ZW01 there are several relatively strong bands in the vibrational frequency range of 1000 $2000 \mathrm{~cm}^{-1}$. The strong band with a calculated anharmonic frequency at $1482 \mathrm{~cm}^{-1}$ may be assigned to the umbrella vibration of the protonated amino group in the zwitterionic lysine moiety. This mode is completely absent from the IRMPD spectrum and would suggest that the protonbound dimer is not zwitterionic under the experimental conditions. The calculated anharmonic bands at 1647 and $1766 \mathrm{~cm}^{-1}$ correspond to the asymmetric stretching $\left(v_{\mathrm{as}}\right)$ vibrations for the carboxylate group and carbonyl group, respectively. Another strong band at $1666 \mathrm{~cm}^{-1}$ is from the asymmetric bending $\left(\delta_{\mathrm{as}}\right)$ vibration of the side chain protonated amino group.

For the second most stable zwitterionic isomer (LLZW02), the strong band at $1747 \mathrm{~cm}^{-1}$ in the anharmonic calculated spectrum is the asymmetric stretching $\left(v_{\text {as }}\right)$ 


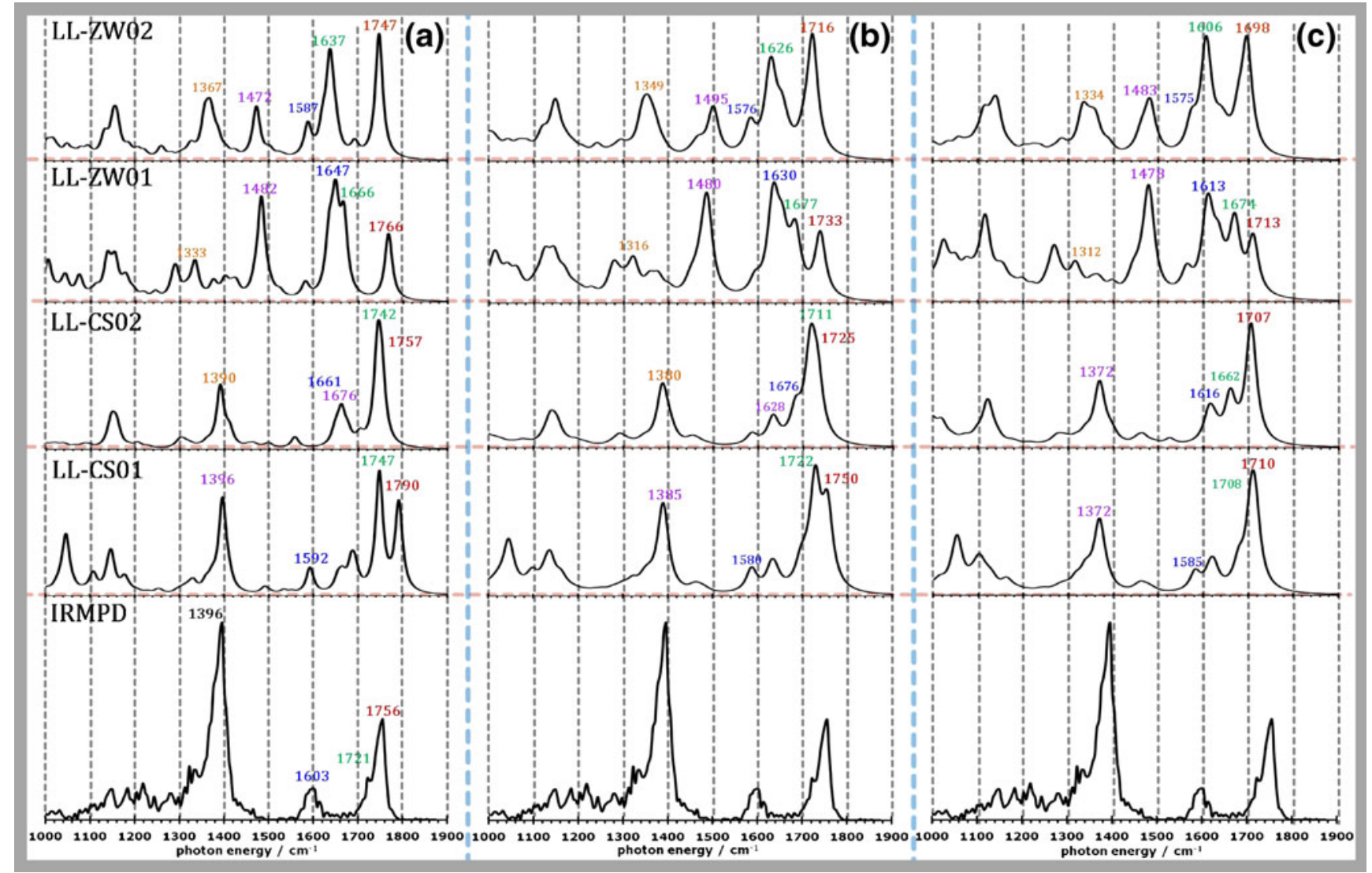

Figure 2. Comparison of the experimental IRMPD spectrum of the proton-bound dimer of lysine with, (a) calculated anharmonic, (b) harmonic (scaled by 0.96) B3LYP/6-31+G(d), and (c) harmonic (scaled by 0.95) MP2/6-31+G(d) spectra. Frequencies are reported in units of wave numbers $\left(\mathrm{cm}^{-1}\right)$

vibration of the carbonyl group, and the corresponding band of the carboxylate group appears at $1637 \mathrm{~cm}^{-1}$. Several bands between these two bands are due to the asymmetric bending $\left(\delta_{\mathrm{as}}\right)$ vibrations of the two $-\mathrm{NH}_{3}$ groups and the symmetric bending $\left(\delta_{\mathrm{s}}\right)$ modes of the $-\mathrm{NH}_{2}$ groups. The band at $1472 \mathrm{~cm}^{-1}$ is from the symmetric bending $\left(\delta_{\mathrm{s}}\right)$ vibration of the protonated $\alpha$-amino group. The band at $1367 \mathrm{~cm}^{-1}$ may be assigned to the symmetric stretch $\left(v_{\text {as }}\right)$ of the carboxylate group.

The calculated spectra of the two zwitterionic isomers are notably different from the experimental IRMPD spectra. According to the results of calculated energetics and spectra, although the existence of the zwitterionic isomers of the proton-bound dimer cannot be completely excluded, it is very clear that the non-zwitterionic isomers (LL-CS01 and LL-CS02) should be the dominant species under the experimental conditions. According to our previous results [20], the zwitterionic structure of glycine is not involved in the glycine proton-bound dimer and the zwitterionic structure of proline does exist as one of the two co-existing most stable forms of proton-bound proline dimer.

Usually there is a trend that the more basic amino acids more easily form zwitterionic structures in complexes. The literature values of the proton affinities of lysine and proline are reported as 996 and $920 \mathrm{~kJ} \mathrm{~mol}^{-1}$, respectively [55]. The gas basicity of lysine is $950 \mathrm{~kJ} \mathrm{~mol}^{-1}$ and that of proline is $886 \mathrm{~kJ} \mathrm{~mol}^{-1}$. According to this trend in proton affinity and gas basicity data, lysine should then more easily form a zwitterionic structure in complexes than does proline. However the experimental and calculated results indicate very clearly that while the zwitterionic structure of the proton-bound dimer of proline dominates [56], the zwitterionic structure of the proton-bound dimer of lysine is not evident under the experimental conditions. As noted above, this difference is due to the complex hydrogen bonding scheme exhibited by the proton-bound dimer of lysine. These results thus demonstrate quite dramatically that the side chain can have a very complex influence on the structures of complexes of protonated amino acids which complicate any trend that might otherwise occur with proton affinity considerations.

\section{Conclusions}

The structure of the proton-bound lysine dimer has been investigated by IRMPD spectroscopy in combination with electronic structure calculations. IRMPD spectra of the protonated lysine dimer have been recorded using the combination of a free electron laser (FEL) and an electrospray-ion trap mass spectrometer. The structures of different 
possible isomers of proton-bound lysine dimer have been optimized at the B3LYP/6-31+G(d), MP2/6-31+G(d), and $\mathrm{MP} 2 / 6-31+\mathrm{G}(\mathrm{d}, \mathrm{p})$ levels of theory. Infrared spectra are reported for both anharmonic and harmonic (scaled by 0.96) frequencies at the $\mathrm{B} 3 \mathrm{LYP} / 6-31+\mathrm{G}(\mathrm{d})$ level of theory, in addition to harmonic (scaled by 0.95 ) frequencies at the MP2/6-31+G(d) level of theory. Comparison of the scaled harmonic and anharmonic B3LYP/6-31+G(d) calculated spectra of isomers LL-CS01 and LL-CS02 with the IRMPD spectrum demonstrates good agreement in both cases. The scaled harmonic and anharmonic B3LYP/6-31+G(d) spectra each have a MAD of $14 \mathrm{~cm}^{-1}$ between the experimental and calculated frequencies. The agreement between the IRMPD spectrum and the scaled harmonic MP2/6-31+G(d) calculated spectrum is less accurate, with a MAD of $26 \mathrm{~cm}^{-1}$, suggesting that both the scaled harmonic and anharmonic B3LYP calculations outperform the MP2 calculation obtained using the $6-31+\mathrm{G}(\mathrm{d})$ basis set. The preceding comparison is very useful since harmonic MP2 calculations are significantly more costly than equivalent harmonic B3LYP calculations. Another important consideration to note is the effectively equal performance of the scaled harmonic B3LYP/6-31+G(d) calculation to that of the anharmonic calculation at the same level of theory. Similar to the benefit of using B3LYP over the MP2 method described above, the scaled harmonic frequencies with the B3LYP method are obtained at substantially lower cost than the equivalent anharmonic calculation. Based on the preceding statement, it is suggested that systems of similar size, charge and functionality to that of the proton-bound lysine dimer should be adequately simulated by calculations at the harmonic B3LYP/6-31+G(d) level of theory with frequencies scaled by 0.96 [51].

LL-CS01 is the most stable isomer based on calculation at the MP2/aug-cc-pVTZ//B3LYP/6-31+G(d) level of theory. In this isomer, the proton is bound to the side chain amino group of one lysine, as in the protonated lysine monomer. This is followed by the formation of one intramolecular hydrogen bond with the $\alpha$-amino nitrogen and two intermolecular hydrogen bonds with the carbonyl oxygen and side chain amino nitrogen of the neutral lysine moiety, respectively, with almost the same bond lengths of $1.88,1.85$, and $1.89 \AA$. LL-ZW01 is the most stable zwitterionic isomer, in which the structure of the protonated lysine moiety is very similar to the protonated lysine monomer. The proton is bound to the amino nitrogen of the side chain, followed by the formation of two intramolecular hydrogen bonds with the $\alpha$-amino nitrogen and carbonyl oxygen and another very strong intermolecular hydrogen bond with the carboxylate group of the neutral zwitterionic lysine with a short bond length of $1.64 \AA$. In the zwitterionic lysine moiety, the protonated $\alpha$-amino group forms two strong intramolecular hydrogen bonds with the adjacent carboxylate and side chain amino group, respectively. Thus, the negatively charged carboxylate group is effectively stabilized by the two positively charged proto- nated amino groups. The experimental IRMPD and calculated spectra demonstrate that the most stable isomer does not involve a zwitterionic structure. According to the common trend in the proton affinity and gas basicity data, lysine should more easily form a zwitterionic structure than proline. However, our experimental and calculated results indicate very clearly that while the zwitterionic structure of proline dominates, the zwitterionic structure of lysine is not evident under the experimental conditions. These results demonstrate that the side chain has a very complex influence on the formation of zwitterionic structure of amino acids. Continued investigation of zwitterionic structure of amino acids in the gas phase will aid in a further understanding of the structure, property, and function of biological molecules.

\section{Acknowledgments}

The authors acknowledge generous financial support by the Natural Sciences and Engineering Research Council of Canada (NSERC) and the financial support of the European Commission through the NEST/ADENTURE program (EPITOPES, project no. 15637). The authors are very grateful for the valuable assistance of the CLIO team, P. Maitre, J. Lemaire, J. M. Bakker, T. Besson, D. Scuderi, and J. M. Ortega. They are also grateful to G. Ohanessian and C. Clavaguéra for assistance with the REMD calculations.

\section{References}

1. Bosshard, H.R., Marti, D.N., Jelesarov, I.: Protein stabilization by salt bridges: concepts, experimental approaches, and clarification of some misunderstandings. J. Mol. Recognit. 17, 1-16 (2004)

2. Dey, M., Cao, C., Sicheri, F., Dever, T.E.: Conserved intermolecular salt bridge required for activation of protein kinases PKR, GCN2, and PERK. J. Biol. Chem. 282, 6653-6660 (2007)

3. Bush, M.F., Oomens, J., Saykally, R.J., Williams, E.R.: Effects of alkaline earth metal ion complexation on amino acid zwitterion stability: results from infrared action spectroscopy. J. Am. Chem. Soc. 130, 6463-6471 (2008)

4. Cerda, B.A., Wesdemiotis, C.: Zwitterionic versus charge-solvated structures in the binding of arginine to alkali metal ions in the gas phase. Analyst 125, 657-660 (2000)

5. Julian, R.R., Hodyss, R., Beauchamp, J.L.: Salt bridge stabilization of charged zwitterionic arginine aggregates in the gas phase. J. Am. Chem. Soc. 123, 3577-3583 (2001)

6. Remko, M., Fitz, D., Rode, B.M.: Effect of metal ions ( $\mathrm{Li}+, \mathrm{Na}+, \mathrm{K}+$, $\mathrm{Mg} 2+, \mathrm{Ca} 2+, \mathrm{Ni} 2+, \mathrm{Cu} 2+$, and $\mathrm{Zn} 2+)$ and water coordination on the structure and properties of L-Arginine and Zwitterionic L-Arginine. $J$. Phys. Chem. A 112, 7652-7661 (2008)

7. Rozman, M.: Theoretical study of the gas-phase structures of sodiated and cesiated leucine and isoleucine: zwitterionic structure disfavored in kinetic method experiments. J. Mass Spectrom. 40, 1357-1361 (2005)

8. Rozman, M., Srzic, D., Klasinc, L.: Gas-phase interaction of protonated lysine with water. Int. J. Mass spectrom. 253, 201-206 (2006)

9. Wu, R.H., McMahon, T.B.: Structures, energetics, and dynamics of gas phase ions studied by FTICR and HPMS. Mass Spectrom. Rev. 28, 546-585 (2009)

10. Wyttenbach, T., Witt, M., Bowers, M.T.: On the question of salt bridges of cationized amino acids in the gas phase: glycine and arginine. Int. J. Mass Spectrom. 182, 243-252 (1999)

11. Wyttenbach, T., Witt, M., Bowers, M.T.: On the stability of amino acid zwitterions in the gas phase: the influence of derivatization, proton affinity, and alkali ion addition. J. Am. Soc. Mass Spectrom. 122, 34583464 (2000)

12. Armentrout, P.B., Armentrout, E.I., Clark, A.A., Cooper, T.E., Stennett, E.M.S., Carl, D.R.: An experimental and theoretical study of alkali 
metal cation interactions with cysteine. J. Phys. Chem. B 114, 39273937 (2010)

13. Bowman, V.N., Heaton, A.L., Armentrout, P.B.: Metal cation dependence of interactions with amino acids: bond energies of $\mathrm{Rb}+$ to Gly, Ser, Thr, and Pro. J. Phys. Chem. B 114, 4107-4114 (2010)

14. Dunbar, R.C., Hopkinson, A.C., Oomens, J., Siu, C.K., Siu, K.W.M., Steill, J.D., Verkerk, U.H., Zhao, J.F.: Conformation switching in gasphase complexes of histidine with alkaline earth ions. J. Phys. Chem. B 113, 10403-10408 (2009)

15. Dunbar, R.C., Steill, J.D., Polfer, N.C., Oomens, J.: Dimeric complexes of tryptophan with M2+ metal ions. J. Phys. Chem. A 113, 845-851 (2009)

16. O'Brien, J.T., Prell, J.S., Steill, J.D., Oomens, J., Williams, E.R.: Changes in binding motif of protonated heterodimers containing valine and amines investigated using IRMPD spectroscopy between 800 and $3700 \mathrm{~cm}(-1)$ and theory. J. Am. Chem. Soc. 131, 3905-3912 (2009)

17. Prell, J.S., O'Brien, J.T., Steill, J.D., Oomens, J., Williams, E.R.: Structures of protonated dipeptides: the role of arginine in stabilizing salt bridges. J. Am. Chem. Soc. 131, 11442-11449 (2009)

18. $\mathrm{Wu}, \mathrm{R}$., McMahon, T.B.: Stabilization of zwitterionic structures of amino acids (Gly, Ala, Val, Leu, Ile, Ser, and Pro) by ammonium ions in the gas phase. J. Am. Chem. Soc. 130, 3065-3078 (2008)

19. $\mathrm{Wu}, \mathrm{R} . \mathrm{H}$. , McMahon, T.B.: Stabilization of the zwitterionic structure of proline by an alkylammonium ion in the gas phase. Angew. Chem. Int Edit. 46, 3668-3671 (2007)

20. Wu, R.H., McMahon, T.B.: Infrared multiple photon dissociation spectra of proline and glycine proton-bound homodimers. Evidence for zwitterionic structure. J. Am. Chem. Soc. 129, 4864 (2007)

21. Kapota, C., Lemaire, J., Maitre, P., Ohanessian, G.: Vibrational signatures of charge solvation versus salt bridge isomers of sodiated amino acids in the gas phases. J. Am. Chem. Soc. 126, 1836-1842 (2004)

22. Bush, M.F., Forbes, M.W., Jockush, R.A., Oomens, J., Polfer, N.C., Saykally, R.J., Williams, E.R.: Infrared spectroscopy of cationized lysine and e-N-Methyllysine in the gas phase: effects of alkali-metal ion size and proton affinity on zwitterion stability. J. Phys. Chem. A 111, 7753-7760 (2007)

23. Lemoff, A.S., Bush, M.F., O'Brien, J.T., Williams, E.R.: Structures of lithiated lysine and structural analogues in the gas phase: effects of water and proton affinity on zwitterionic stability. J. Phys. Chem. A 110, 8433-8442 (2006)

24. Leng, Y.M., Zhang, M.L., Song, C., Chen, M.L., Lin, Z.J.: A semiempirical and $\mathrm{Ab}$ Initio combined approach for the full conformational searches of gaseous lysine and lysine- $\mathrm{H}_{2} \mathrm{O}$ complex. THEOCHEM J. Mol. Struct. 858, 52-65 (2008)

25. Lemaire, J., Boissel, P., Heninger, M., Mauclaire, G., Bellec, G., Mestdagh, H., Simon, A., Caer, S.L., Ortega, J.M., Glotin, F., Maitre, P.: Gas phase infrared spectroscopy of selectively prepared ions. Phys. Rev. Lett. 89, 273001-273004 (2002)

26. Maitre, P., Le Caer, S., Simon, A., Jones, W., Lemaire, J., Mestdagh, H. N., Heninger, M., Mauclaire, G., Boissel, P., Prazeres, R., Glotin, F., Ortega, J.M.: Ultrasensitive spectroscopy of ionic reactive intermediates in the gas phase performed with the first coupling of an IR FEL with an FTICR-MS. Nucl. Instrum. Meths. Phys. Res. A 507, 541-546 (2003)

27. Wu, R.H., McMahon, T.B.: IRMPD spectra of gly center Dot NH4+ and proton-bound betaine dimer: evidence for the smallest gas phase zwitterionic structures. J. Mass Spectrom. 43, 1641-1648 (2008)

28. Penev, E.S., Lampoudi, S., Shea, J.E.: TiREX: replica-exchange molecular dynamics using TINKER. Comput. Phys. Commun. 180, 2013-2019 (2009)

29. Ponder, J. W. TINKER, Software Tools for Molecular Design, Version 4.22004.

30. Semrouni, D., Balaj, O.P., Calvo, F., Correia, C.F., Clavaguera, C., Ohanessian, G.: structure of sodiated octa-glycine: IRMPD spectroscopy and molecular modeling. J. Am. Soc. Mass Spectrom. 21, 728$738(2010)$

31. Frisch, M. J.; Trucks, G. W.; Schlegel, H. B.; Scuseria, G. E.; Robb, M. A.; Cheeseman, J. R.; Scalmani, G.; Barone, V.; Mennucci, B.; Petersson, G. A.; Nakatsuji, H.; Caricato, M.; Li, X.; Hratchian, H. P.; Izmaylov, A. F.; Bloino, J.; Zheng, G.; Sonnenberg, J. L.; Hada, M.; Ehara, M.; Toyota, K.; Fukuda, R.; Hasegawa, J.; Ishida, M.; Nakajima, T.; Honda, Y.; Kitao, O.; Nakai, H.; Vreven, T.; Montgomery, J. A.; Peralata, J. E.; Ogliaro, F.; Bearpark, M.; Heyd, J. J.; Brothers, E.; Kudin, K. N.; Staroverov, V. N.; Kobayashi, R.; Normand, J.;
Raghavachari, K.; Rendell, A.; Burant, J. C.; Iyengar, S. S.; Tomasi, J.; Cossi, M.; Rega, N.; Millam, J. M.; Klene, M.; Knox, J. E.;; Cross, J. B.; Bakken, V.; Adamo, C.; Jaramillo, J.; Gomperts, R.; Stratmann, R. E.; Yazyev, O.; Austin, A. J.; Cammi, R.; Pomelli, C.; Ochterski, J. W.; Martin, R. L.; Morokuma, K.; Zakrzewski, V. G.; Voth, G. A.; Salvador, P.; Dannenberg, J. J.; Dapprich, S.; Daniels, A. D.; Farkas, O.; Foresman, J. B.; Ortiz, J. V.; Cioslowski, J.; Fox D. J. Gaussian 09, Revision A.02. Gaussian, Inc.: Wallingford CT 2009.

32. Gil, A., Simon, S., Rodriguez-Santiago, L., Bertran, J., Sodupe, M.: Influence of the side chain in the structure and fragmentation of amino acids radical cations. J. Chem. Theory Comput. 3, 2210-2220 (2007)

33. Noguera, M., Sodupe, M., Bertran, J.: Effects of protonation on protontransfer processes in guanine-cytosine watson-crick base pairs. Theor. Chem. Acc. 112, 318-326 (2004)

34. Topol, I.A., Burt, S.K., Russo, N., Toscano, M.: Theoretical calculations of glycine and alanine gas-phase acidities. J. Am. Soc. Mass Spectrom. 10, 318-322 (1999)

35. Wu, R., McMahon, T.B.: Infrared multiple photon dissociation spectroscopy as structural confirmation for GlyGlyGlyH ${ }^{+}$and AlaAlaAlaH ${ }^{+}$in the gas phase. Evidence for amide oxygen as the protonation site. J. Am. Chem. Soc. 129, 11312-11313 (2007)

36. $\mathrm{Wu}, \mathrm{R} . \mathrm{H} ., \mathrm{McMahon}, \mathrm{T} . \mathrm{B}$.: Investigation of cation $-\pi$ interactions in biological systems. J. Am. Chem. Soc. 130, 12554 (2008)

37. Marta, R.A., Wu, R., Eldridge, K.R., Martens, J.K., McMahon, T.B.: Infrared vibrational spectra as a structural probe of gaseous ions formed by caffeine and theophylline. Phys. Chem. Chem. Phys. 12, 3431-3442 (2010)

38. Marta, R.A., Wu, R., Eldridge, K.R., Martens, J.K., McMahon, T.B.: The sodium cation-bound dimer of theophylline: IRMPD spectroscopy of a highly symmetric electrostatically bound species. Int. J. Mass spectrom. 297, 76-84 (2010)

39. Balaj, O.P., Kapota, C., Lemaire, J., Ohanessian, G.: Vibrational signatures of sodiated oligopeptides (GG-Na+, GGG-Na+, AA-Na+, and AAA-Na+) in the gas phase. Int. J. Mass spectrom. 269, 196-209 (2008)

40. Bauschlicher, J.C.W., Langhoff, S.R., Partridge, H., Rice, J.E., Komornicki, A.: A Theoretical Study of $\mathrm{Na}(\mathrm{H} 2 \mathrm{O}) n+(n=1-4)$. J. Chem. Phys. 95, 5142-5148 (1991)

41. Bytheway, I., Wong, M.W.: The prediction of vibrational frequencies of iinorganic molecules using density functional theory. Chem. Phys. Lett. 282, 219-226 (1998)

42. Correia, C.F., Clavaguera, C., Erlekam, U., Scuderi, D., Ohanessian, G.: IRMPD spectroscopy of a protonated, phosphorylated dipeptide. Chem. Phys. Chem. 9, 2564-2573 (2008)

43. Halls, M.D., Velkovski, J., Schlegel, H.B.: Harmonic frequency scaling factors for Hartree-Fock, S-VWN, B-LYP, B3-LYP, B3-PW91, and MP2 with the Sadlej pVTZ electric property basis set. Theor. Chem. Acc. 105, 413-421 (2001)

44. Jaramillo, J., Scuseria, G.E.: Performance of a kinetic energy densitydependent functional (VSXC) for predicting vibrational frequencies. Chem. Phys. Lett. 312, 269-276 (1999)

45. Johnson, B.G., Gill, P.M.W., Pople, J.A.: The performance of a family of density functional methods. J. Chem. Phys. 98, 5612-5626 (1993)

46. Scott, A.P., Radom, L.: Harmonic vibrational frequencies: an evaluation of Hartree-Fock, Moller-Plesset, quadratic configuration interaction, density functional theory, and semiempirical scale factors. J. Phys. Chem. 100, 16502-16513 (1996)

47. Scuderi, D., Correia, C.F., Balaj, O.P., Ohanessian, G., Lemaire, J., Maitre, P.: Structural characterization by IRMPD spectroscopy and DFT calculations of deprotonated phosphorylated amino acids in the gas phase. Chem. Phys. Chem. 10, 1630-1641 (2009)

48. Barone, V.: Vibrational zero-point energies and thermodynamic functions beyond the harmonic approximation. J. Chem. Phys. 120, 3059-3065 (2004)

49. Friesner, R.A.: Ab Initio quantum chemistry: methodology and applications. Proc. Natl. Acad. Sci. U.S.A. 102, 6648-6653 (2005)

50. Dunning Jr., T.H.: Gaussian basis sets for use in correlated molecular calculations. i. the atoms boron through neon and hydrogen. J. Chem. Phys. 90, 1007-1023 (1989)

51. Merrick, J.P., Moran, D., Radom, L.: An evaluation of harmonic vibrational frequency scale factors. J. Phys. Chem. A 111, 11683-11700 (2007) 
52. von Helden, G., van Heijnsbergen, D., Meijer, G.: Resonant ionization using IR light: a new tool to study the spectroscopy and dynamics of gas-phase molecules and clusters. J. Phys. Chem. A 107, 1671-1688 (2003)

53. Asmis, K.R., Pivonka, N.L., Santambrogio, G., Brummer, M.K.C., Neumark, D.M., Woste, L.: Gas-phase infrared spectrum of the protonated water dimer. Science 299, 1375-1377 (2003)

54. Polfer, N.C., Oomens, J., Moore, D.T., von Helden, G., Meijer, G., Dunbar, R.C.: Infrared spectroscopy of phenylalanine $\mathrm{Ag}(\mathrm{I})$ and $\mathrm{Zn}$
(II) complexes in the gas phase. J. Am. Chem. Soc. 128, 517-525 (2006)

55. Lias, S.G., Hunter, E.P.: Evaluated gas phase basicities and proton affinities of molecules: an update. J. Phys. Chem. Ref. Data 27, 413656 (1998)

56. Wu, R., McMahon, T.B.: Infrared multiple photon dissociation spectra of proline and glycine proton-bound homodimers. Evidence for zwitterionic structure. J. Am. Chem. Soc. 129, 4864-4865 (2007) 